\title{
Node-Disjoint On-demand Multipath Routing with Route Utilization in Ad-Hoc Networks
}

\author{
Mona Gupta \\ Student- M.E
}

\author{
Neeraj Kumar \\ Assistant Professor
}

\author{
CSED, Thapar UniversityCSED, Thapar University \\ Patiala, India Patiala, India
}

\begin{abstract}
Mobile Ad Hoc Network (MANET) is a dynamic wireless network without any pre-existing infrastructure and each node therefore operate in a self-organized and decentralized manner, acting as a router. Multipath routing in MANETis a challenging problem. In this paper, an attempt has been made to compare the accomplishment of conspicuous ondemand routing protocols for mobile ad hoc networks. There are many routing protocols such as ad hoc on demand distance vector routing (AODV), ad hoc on demand multipath distance vector protocol (AOMDV) that have been used. The constant change in network topology and the inherently unreliable available paths make the multipath routing difficult.The paper presents the nodedisjoint multipath routing protocol that allows the establishment of multiple paths between the unique source and destination and thus improving end-to-end delay, routing overhead and packet delivery ratio. The path selection algorithm for MANET embraces the entropy metric in order to find the stability of route and obtain an optimum path.
\end{abstract}

\section{Keywords}

MANET; multipath routing; on demand routing, entropy

\section{INTRODUCTION}

A MANET [1] is a collection of mobile nodes which act as both a host and a router, and thus exchange data dynamically without relying on any fixed base station. The transmissions of packets take place via the intermediate nodes within a specified range that does certain functions on its received data. The application areas of MANET include emergency search, battlefield, acquiring data and rescue sites in distant areas, sharing data dynamically in conventions and classrooms by mobile computing devices [2].

A path between two mobile nodes in MANET can be made by either one or multiple hops. Therefore, there could be many changes in the network topology caused due to the mobility of the mobile nodes. Thus it becomes important to choose the routes wisely. In order to perform this task efficiently, there are many on-demand routing protocols that have been developed. Many existing on-demand routing protocols build and use one pathbetween a pair of source and destination; therefore the route may become temporarily unavailable or invalid due to the node mobility, node failure, and the dynamic features of the channel. It increases the efforts of finding an alternative path and delay in packet delivery. The problem is addressed by multipath routing that provides more than one path between a pair of source and destination. Finding or computing multiple paths in one route discovery attempt by multipath routing protocols, try to overcome these problems. Duplicate paths can be formed at both traffic sources and intermediate nodes. New routes are discovered when all the paths fail. Thus it decreases the latency involved in finding routes and transmitting packets. By providing data packets on multiple paths at the same time load balancing can also be done[3]. Along with on-demand multipath routing, the node-disjoint paths are used as it is the strongest measure of path independence. Node-disjoint paths are those paths which do not have any common node, except the source and destination and hence they do not have any link in common. By distributing traffic among a set of nodedisjoint paths route failure protection is provided. The paper tries to improve the performance of AODV by introducing multipath routing and reducing its routing packets.

The objective of this paper is to describe the node-disjoint on-demand multipath routing protocols and show the path selection entropy for a MANET. The paper is organized as follows: Section 2 gives an overview of routing protocols of on-demand ad hoc networks. In section 3 theoretical frameworks is introduced. Section 4 discusses the route discovery process. Finally the paper concludes in section 5.

\section{ROUTING PROTOCOLS}

In this section we provide the useful details of various routing protocols in wireless ad hoc networks that facilitate the on-demand routing.

\subsection{AODV protocol description}

AODV [4] routing protocol is a reactive, stateless, single path on-demand routing protocol for a MANET. The authors of AODV call it a pure on-demand routing protocol because the mobile nodes that are not on a selected path neither keep any routing information nor share any routing table information [5]. The protocol works by:

- Discovering a route.

- Maintaining a route.

When a node (source) wants to send data to another node (destination), the route discovery phase is started to find the other node. The path request packet (PRQ) is broadcasted with a unique PID to all its neighbors. The broad cast is 
done till a sink is found or a node with new path to sink is known.

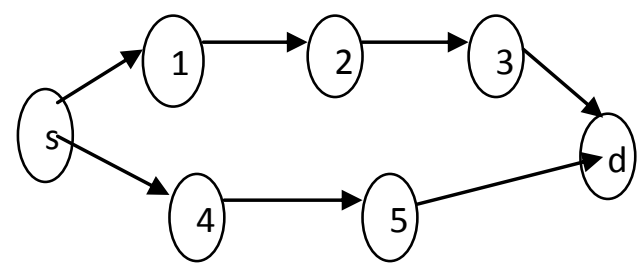

Fig1: The PRQ packet broadcast

All the neighbors make an entry in their routing table for the given PID. The path reply packet (PRP) is created when the destination node or an intermediate node with an acceptable route to destination has got the PRQ. The PRP will be a unicast in the reverse direction. When PRP is routed in reverse direction, nodes in this direction set up a forward direction entry in their routing tables that point to a node from which PRP came.

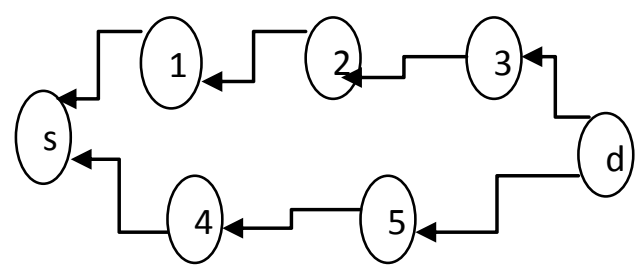

Fig2: Destination returning PRP packet

Fig 1 shows the broadcast of PRQ packet from the source while Fig 2 shows the unicast of PRP packet from the destination. There is another packet called the path defect packet (PDF). When a mobile node moves away, a link failure may happen in between the two nodes, the PDF packet is broadcasted in that case. The source may have to re-initiate discovering route to the desired destination. A HELLO message is exchanged in between the neighboring mobile nodes in order to give the information related to link status.

The AODV protocol decreases the number of required broadcasts by creating the routes only on on-demand basis. Due to the power limitations, the routing protocol is not suitable for ad hoc sensor networks [6].

\subsection{AOMDV protocol description}

AOMDV [7] is an extension of AODV protocol. To create multiple node-disjoint and loop free routes during the route discovery process, the AOMDV is derived. Thus the protocol consists of two major parts:

- $\quad$ To find node-disjoint routes.

- $\quad$ To create and maintain multiple loop-free routes. In each PRQ, respectively PRP coming at a node defines an alternate route to the source or destination. It could then lead to looping of routes. In order to avoid such looping, hopcount is made. The hopcounth of a node for a destination is the maximum count of hops for multiple paths available at that node. For each destination, routing entry consist of next-hop and hopcount. The sequence number for all the next hops is the same. This leads to maintenance of multiple loop-free routes.

In AOMDV, duplicate copies of PRQ are not disposed immediately. Each of the packets is checked for a nodedisjoint path to the source. All the PRQs should come via different neighbors of the source in order to get nodedisjoint paths. However, at the destination different criteria is used, the routes determined there are link-disjoint, not node-disjoint. Here, the PRQs should come via unique neighbors [8].

The encapsulated features of AOMDV therefore includes absence of entire path information at source, discovery of multiple routes in single process, maintenance of next-hop for destinations, looping avoided via maximum hopcount and creation of multiple link-disjoint routes. The decreased normal end-to-end delay, high data throughput, and controlled overhead indicates the protocol as an improvement over AODV [9].

\subsection{AODVM protocol description}

AODVM [10] is the multipath extension of AODV routing protocol for MANET. The route with lower number of common nodes is chosen to be link-disjoint. Here, the intermediate nodes cannot send PRP straightforward to the source. The nodes cannot dispose of the redundant PRQs and therefore a record of coming PRQs is maintained in a PRQ table. For all the incoming PRQs, destination replies with a PRP. The nodes in between sends this PRP to the next node according to the PRQ table on a selected path. A node is removed from PRQ table if it broadcasts PRQ and its neighbor hears it. Therefore, a node is engaged in only one route and hence the routes are confirmed to be nodedisjoint. While the discovery of a path, it sets up a path using the PRP coming first. The substitute paths are formed by late redundant PRPs. Due to the restriction of hopcount based approach, incapable routes may occur in AODVM.

\subsection{Split Multipath Routing (SMR) and Multipath Source Routing (MSR)}

SMR [11] protocol is a mean to find maximally disjoint paths (node-disjoint paths). It is an example to dynamic source routing (DSR) protocol, where the packets carry their routing information in their headers. The on-demand discovery of routes with least delay is done. The length of such routes may be different. In SMR, the source routing packets consists of entire routes, therefore the source contains the full route information. A unique sequence number and a source ID is contained in each PRQ. All the redundant PRQ packets that have travelled by a unique incoming link are sent by the intermediate nodes. The shortest-delay path or the rapid paths are answered first by the destination. Thus the simultaneous transfer of data over multiple paths is examined.

The MSR [12] protocol is an on-demand reactive protocol that distributes the traffic to multiple routes in a network. It is also an extension to Dynamic Source Routing protocol. Here, a route represents the packets sent at the same time on that path. In order to choose multiple paths, a unique index is maintained for each path in a cache. For path independence disjoint paths are used and the packet headers contain the route information (avoiding loops). 
There are many other multipath routing protocols for MANET like multipath on-demand routing (MOR)[13] that depends on network floods or flooding broadcasts to discover fresh routes, caching and multipath routing protocol (CHAMP)[14] that uses data caching and reduces packet loss by shortest multipath routing,multiple next hop routing protocol $(\mathrm{MNH})$ [15] where intermediate nodes find a new route in case of a link failure, multipath dynamic source routing protocol (MP-DSR)[16] which is fully distributed quality of service (QoS) aware protocol that find multiple node-disjoint paths for data transmission.

Table 1. Multipath Routing Protocols [2]

\begin{tabular}{|l|l|l|l|}
\hline Property & AODVM & AOMDV & $\begin{array}{l}\text { SMR and } \\
\text { MSR }\end{array}$ \\
\hline Routing type & reactive & reactive & reactive \\
\hline $\begin{array}{l}\text { Complete } \\
\text { multiple paths }\end{array}$ & yes & yes & yes \\
\hline $\begin{array}{l}\text { Node-disjoint } \\
\text { paths }\end{array}$ & no & no & yes \\
\hline $\begin{array}{l}\text { Loop-free } \\
\text { paths }\end{array}$ & yes & yes & yes \\
\hline $\begin{array}{l}\text { Complete } \\
\text { routes known }\end{array}$ & no & no & yes \\
\hline TTL limitation & yes & yes & yes \\
\hline $\begin{array}{l}\text { Common clock } \\
\text { needed }\end{array}$ & no & no & no \\
\hline QoS Support & no & no & no \\
\hline $\begin{array}{l}\text { Multicast } \\
\text { Support }\end{array}$ & no & no & no \\
\hline $\begin{array}{l}\text { Simultaneous } \\
\text { Paths }\end{array}$ & no & no & yes \\
\hline $\begin{array}{l}\text { Security } \\
\text { Support }\end{array}$ & no & no & no \\
\hline $\begin{array}{l}\text { Power } \\
\text { Management }\end{array}$ & no & no & no \\
\hline $\begin{array}{l}\text { Implementatio } \\
\text { n }\end{array}$ & AODV & AODV & DSR \\
\hline
\end{tabular}

\section{THEORATICAL FRAMEWORK}

A MANET can be seen as a flow network (Fig 3) with the communication pair $(t, r)$ in where $t$ is the transmitter that wants to send its piece of information to the receiver $r$ via a set of intermediate nodes present in the network. Now, the problem of finding optimum path between source $t$ and destination $r$ is similar to finding minimum cost spanning tree for a graph with $\mathrm{n}$ nodes.

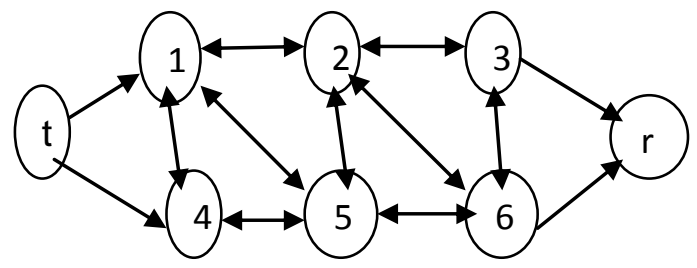

Fig 3: The PRQ packet broadcast

There are algorithms like Prims and kruskals [17] that can be used to find minimum cost spanning tree in a MANET flow network and therefore optimizing the task of determining multiple paths. The parameter cost is replaced with the metric entropy which is a measure of disorder or uncertainty in a system. In this paper we will discuss the multiple path utilization procedure.

\section{MULTIPLE ROUTE DISCOVERYPROCEDURE}

The description of on-demand multiple routing protocols along with the entropy of path selection for accomplish this, the AODV packets PRQ, PRP, PDF and HELLO are used and three route discovery procedures are undertaken:

- Discovery of route.

- Utilization of route.

- Maintenance of route.

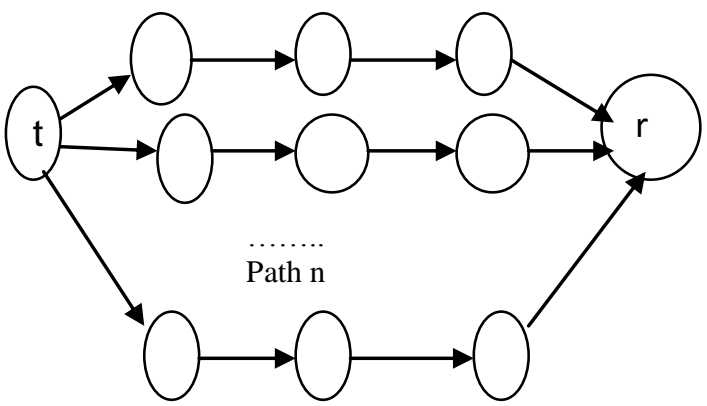

Fig 4:Discovering multiple routes

\subsection{Route Discovery Procedure}

The route discovery procedure is same as introducing multiple routes in ad hoc on-demand distance vector multiple routing protocol. In this process, the source takes and accepts the first PRP packet (coming from the destination along with other PRP packets) without and imposed conditions and the corresponding route is accepted. A measure of the delay in transmission is applied to carry out the procedure of selecting a forward route. A packet is accepted as a fresh forward path, when the time taken to send the redundant packet is same or fresh than the previous time entry in the routing table. This procedure of route discovery is not capable of finding node-disjoint or link-disjoint paths, that intermediate nodes do not select a route of common nodes.

The procedure balances the load and prevents frequent discoveries. It works same as AODV protocol where PRQ is broadcasted by the source initially and the corresponding routes are recorded in the routing table. If a node gets a delayed PRQ packet, it confirms the source route list and the packets are disposed of if a routing loop occurs.

In this procedure, we use entropy [18] to enhance AODV routing protocol by adding nodes' entropy in PRQ and PRP packets of AOMDV.

\subsection{Entropy based route Utilization Procedure}

Entropy is the measure of amount of information and noise present in a signal. In the current discussion, it represents the measure of disturbance and ambiguity in a network. In 
order to choose the best feasible path from the source to the destination, where there are multiple paths being established, we can use some common features of the nodes like the entropy parameter and uncertainty in the areas in MANET [18].

In the following, we consider a MANET and associate a node $\mathrm{k}$ and its neighbor 1 with a set of changing characteristics denoted by $C_{k, l}$ (two nodes are neighbors if one could reach other in one hop i.e. direct communication). $C_{k, l}$ is the measure of rapidity or relative speed (velocity vector), relative location (position vector), and bandwidth (rate of delivery of packets). Therefore, any change in the system is reflected as the change in the variable value $C_{k, l}$. The change in $C_{k, l}$ in course of time t is:

$$
C_{k, l}(t) \rightarrow C_{k, l}(t+d t) C_{k, l}
$$

We assume the neighboring mobile nodes in MANET to periodically exchange with each other the mobility information and other local information like node identification (node ID). Let us denote the rapidity or relative speed of node $\mathrm{k}$ at time $\mathrm{t}$ by $r(k, t)$ and of node 1 by $r(l, t)$. Thus, the relative rapidity or relative velocity between two nodes becomes:

$$
r(k, l, t)=r(k, t)-r(l, t)
$$

Similarly, let $p(k, l, t)$ denote the relative position of two nodes $\mathrm{k}$ and 1 at time $\mathrm{t}$ and $b(k, l, t)$ denote the relative bandwidth of two nodes at time t.

The relative mobility for a pair of nodes $(k, l)$ during a time interval $d t$ is stated as their absolute relative speed, location and bandwidth. Now we can define $C_{k, l}$ as:

$$
\frac{\frac{1}{n} \sum_{i=1}^{n}\left|p(k, l, t)+(r(k, l, t)+b(k, l, t)) d t_{i}\right|-\mid p\left(k, l, t_{i+1} \mid\right.}{N}
$$

...(1).

Where $\mathrm{N}$ represents radio range of nodes and $\mathrm{n}$ is the number of unique times $t_{i}$ we intend to calculate velocity information and spread this information to the neighboring mobile nodes within the time interval $d t$.

Now we are in a situation to define entropy $E_{k}(t, d t)$

during time interval $d t$, the entropy could be calculated within all the neighboring nodes or for only a subset of neighbors.

$$
\begin{aligned}
& E_{k}(t, d t)=-\frac{\sum_{j \in D_{k}} Q_{j}(t, d t) \log Q_{j}(t, d t)}{\log P\left(D_{k}\right)} .(2) \\
& \text { and } Q_{j}(t, d t)=\frac{C_{k, j}}{\sum_{i \in D_{k}} C_{k, i}}
\end{aligned}
$$

where $D_{k}$ is the set of neighboring nodes of node k and $P\left(D_{k}\right)$ is the power or cardinality of set $D_{k}$.If the stability of local network need to be calculated, then $D_{k}$ is set of all neighbors of node $\mathrm{k}$ and for the stability of a part of some route, value of $D_{k}$ is neighboring nodes over that route.As observed, the entropy could be normalized so that: $0 \leq E_{k}(t, d t) \leq 1$.

Note: In a given region, if change of variable values is large, entropy is less and if change of variable values is small, entropy is more.

Let the route stability $\mathrm{R}$ during some time interval $d t$ between a source node $\mathrm{s}$ and a destination node $\mathrm{d}$ is $R_{s, d}(t, d t)$. In order to find end to end route stability, two measures are undertaken,

$$
\begin{aligned}
& \alpha=R_{s, d}^{1}(t, d t) \text { and } \beta=R_{s, d}^{2}(t, d t), \text { where } \\
& \alpha=\prod_{i=1}^{I_{r}}\left[E_{i}(t, d t)\right] \\
& \text { and } \\
& \beta=\min _{i=\left[1,2,3, \ldots I_{r}\right]}\left[E_{i}(t, d t)\right]
\end{aligned}
$$

Where $I_{r}$ is the number of intermediate mobile nodes between source $\mathrm{s}$ and destination $\mathrm{d}$. The route stability and availability could be estimated by the parameter $\alpha(\beta)$ .Therefore, a route is available between two nodes when $\alpha(\beta)$ is large and not available (or if available it is unstable) when $\alpha(\beta)$ is small.

4.3 Route maintenance procedureBattery drainage, congestion and fading effects may lead to broken routes which may interrupt the routing [19]. A route-error packet is transmitted in the event of failure of link or an intermediate node moving out of range. When a route-error packet is received from a node, the route becomes invalid via that link and switches to some other alternative path. Therefore, the node which does not contain the next node of one that sent a route error packet, the source node now chooses that alternative path.

\subsection{Heuristic Algorithm}

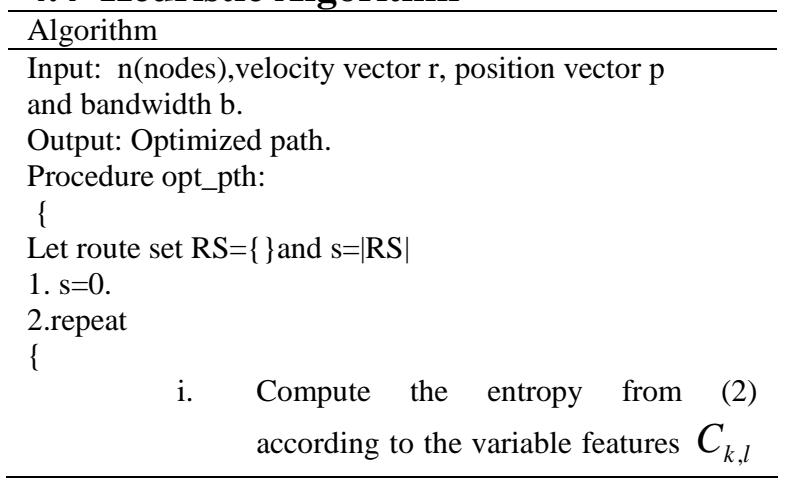




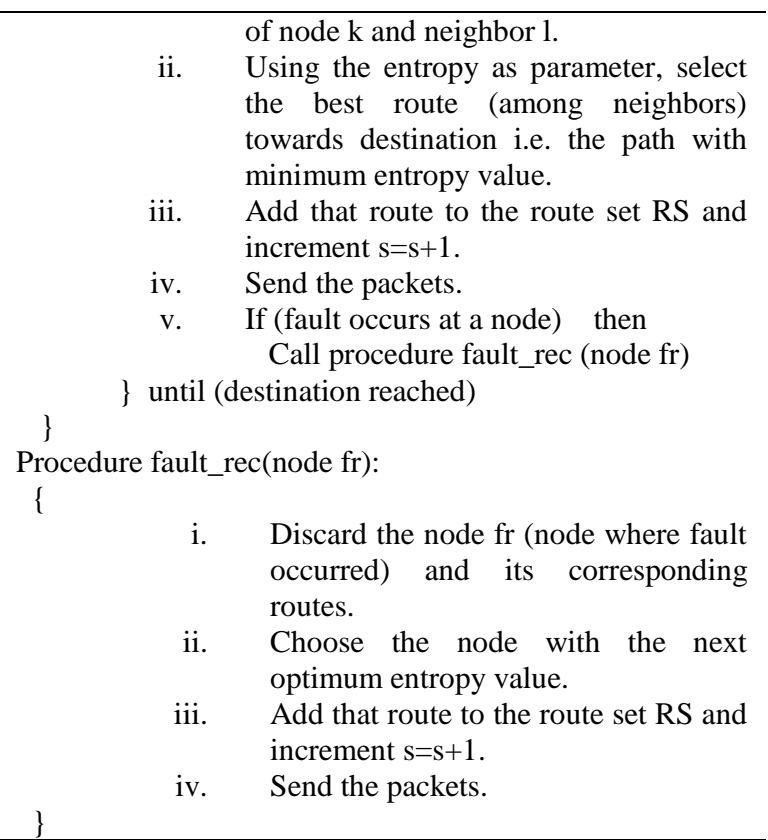

\section{CONCLUSIONS}

The paper proposed an effective approach in making decision in on-demand multipath routing. In order to improve the end-to-end delay, packet delivery ratio and routing overhead, the position vector, velocity vector and bandwidth parameters are taken into account. The paper also compared the performance of some of the multipath on-demand routing protocols which helps in decreasing the overhead of routing using multiple routes. Therefore, the proposed concepts and approaches help in providing a flexible solution to the challenge of maintaining the stability and accuracy of routes in wireless ad hoc networks.

\section{REFERENCES}

[1] IETF Mobile Ad-hoc Networks (MANET) Working Group, http://www.ietf.org/

[2] Sun Baolin, Gui Chao, Zhang Qifei, Yan Bing, Liu Wei,"A Multipath on-Demand Routing with Path Selection Entropy for Ad Hoc Networks", Young Computer Scientists, 2008. ICYCS 2008.

[3] M. R. Pearlman, Z. J. Hass, P. Scholander, S.S. Tabrizi, "On the impact of alternate path routing for load balancing in mobile ad hoc networks," Proceedings of IEEE/ACM MobiHoc 2000, Boston, August 2000, pp. 3-10.

[4] C. E. Perkins, E. M. Royer, "Ad hoc On-Demand Distance Vector Routing (AODV)”, IETF RFC 3561,003

[5] C. E. Perkins et al.: "Ad Hoc on Demand Distance Vector (AODV) Routing", RFC 3561, July.2003.

[6] XiuliRen, HaibinYu,"A novel multipath disjoint routing to support ad hoc wireless sensor networks",Object and Component-Oriented Real-Time distributed Computing, 2006. ISORC 2006.
[7] M. Marina and S. Das, "On-demand Multipath Distance Vector Routing in Ad Hoc Networks", in Proceedings of the International Conference for Network Protocols (ICNP), Riverside, Nov. 2001.

[8] M. K. Marina and S. R. Das, "On-Demand Multipath Distance Vector Routing in Ad hoc Networks", In Proceedings of the Ninth International Conference on Network Protocols (ICNP), California, IEEE Computer Society Press, 11-14 Nov, 2001, pp. 14-23.

[9] M. K. Marina, S. R. Das, "On-demand multipath distance vector routing in ad hoc networks," Proceedings of the 9th IEEE International Conference on Network Protocols (ICNP), 2001, pp. 14-23.

[10] S. Motegi, K. Yoshihara, H. Horiuchi, "Proposal on Multipath Routing for Ad hoc Networks", IEIC Technical Report, 2002, 102(441): 51-56, IN2002125, Nov. 2002 (in Japanese).

[11] S. Lee and M. Gerla, "Split multipath routing with maximally disjoint paths in ad hoc networks", Proceedings of the IEEE ICC, pp. 3201-3205, June 2001.

[12] L. Wang, Y. Shu, M. Dong, L. Zhang and O. Yang, "Adaptive Multipath Source Routing in Ad Hoc Networks", IEEE ICC 2001, Page(s): 867 -871 vol.3, June 2001.

[13] Jing Fang, Bhuvaneswaran R.S., Katayamal Yoshiaki, Takahashi Naohisa,"multipath routing selection strategies in wireless mobile ad hoc networks", Signal Processing, communications and Networking,ICSCN'07.

[14] A. Valera, W. Seah, and S. Rao, "Cooperative Packet Caching and Shortest Multipath Routing in Mobile Ad hoc Networks", INFOCOM 2003, San Francisco, CA, USA, 2003.

[15] Jiang Ming-Hong, Jan Rong-Hong, "An efficient multiple path routing protocol for ad hoc networks", Information Networking Proceedings/ICOIN,2001.

[16] R. Leung, J. Liu, E. Poon, A. Chan and B. Li, "MPDSR: A QoS-Aware Multi-Path Dynamic Source Routing Protocol for Wireless Ad-Hoc Networks", In Proc. of the 26th IEEE Annual Conference on Local Computer Networks (LCN 2001), pp. 132-141, November, 2001

[17] T. H. Cormen, C. E. Leiserson, R. L. Rivest, C. Stein, .Introduction to Algorithms., The MIT Press ,2001.

[18] B. An, and S. Papavassiliou, "An Entropy-Based Model for Supporting and Evaluating Route Stability in Mobile Ad hoc Wireless Networks", IEEE Communications Letters, 2002, 6(8): 328-330.

[19] VahediShabnam, Mohseni Maryam, DarehshoorzadehAmir,"Design a multi-path routing algorithm in ad hoc networks in order to improve fault tolerance",Personal indoor and Mobile Radio Communications, PIMRC 2007. 